\title{
Spectral Transmissive Properties of Five Selected Optical Glasses*
}

\author{
Harry J. Keegan, Marion A. Belknap, and Dorothy J. Cordrey ${ }^{1}$
}

\author{
Measurements of the spectral transmittance and internal transmittance of five optical \\ glasses for the wavelength region 200 to 1,000 millimicrons are reported.
}

\section{Introduction}

The transmittance, $T$, of a plate is defined as the ratio of radiant flux $\left(P_{2}\right)$ transmitted by the plate to the flux $\left(P_{1}\right)$ incident upon it. Internal transmittance $\left(T_{i}\right)$ is defined as the ratio of the flux $(P)$ arriving at the second surface of the plate to that flux $\left(P_{0}\right)$ penetrating the first surface. All values of $T$ and $T_{i}$ are functions of wavelength.

The transmittances and internal transmittances reported were determined for glasses of the NBS melts for which the chemical compositions are given in Research Paper 2504.

The samples were prepared in the NBS Optical Shop in the form of eylinders or plates, $30 \mathrm{~mm}$ in diameter, with surfaces plane, parallel, and polished. The rare earth glass, $n=1.673{ }^{2} \quad \nu=56.2$, was prepared in thicknesses of 5 and $105 \mathrm{~mm}$. The other four glasses were prepared in a thickness of $1 \mathrm{~mm}$ in addition to greater thicknesses. The thin samples were compared against a blank beam, and spectral transmittance was so obtained. The thick samples were compared to the thin sample of the same kind of glass, the resulting ratios being taken as internal transmittance for a sample of thickness equal to the difference between thick and thin specimens. ${ }^{3}$

\section{Spectrophotometric Measurements}

Most spectrophotometers are designed primarily for samples between 0 - and 10- to 50-mm thickness, but can be used with samples of somewhat greater thickness. The greater the thickness, however, the more the instrument is subject to errors resulting from refraction of the various rays in the beam, for although these rays are approximately parallel and at right angles to the surfaces, they are in general not strictly so. The result is, in effect, a change in $P_{1}$ to $P_{0}$ of the above defining relations for transmittance, $P_{2} / P_{1}$, or for internal transmittance, $P / P_{0}$. This is particularly true when, as in the present \footnotetext{
*The companion papers in this issue present values of the index of refraction,
densities, and coefficients of linear thermal expansion for five different type densities, and coefficients of linear thermal expansion for five different types
of optical glasses produced by the Optical Glass Section of the National Burean of optical glasses produced by the Optical Glass Section of the National Bureau
of Standards. These measurements have been made in the different organiof Standards. These measurements have been made in the different organi-
zational parts of the Bureau appropriate to each type of measurement, and are, therefore, presented separately. For each type of glass the samples have been taken from the same melt, and, therefore, these papers present, collectively, a consistent set of data for the five glasses studied.

1 Now Mrs. Frederick J. Does, E. I. duPont de Nemours \& Co., Inc., Parlin, N. J.

${ }_{2} n=$ index of refraction for the sodium $D$ lines, and $\nu$, the Abbe value $=(n-1)$ $\left(n_{P}-n_{C}\right)$, where $n_{F}$ and $n_{\mathrm{C}}$ are refractive indices for the hydrogen $F$ and $C$ lines, respectively.

3 Glass D F , $n=1.649, \nu=33.5$ was prepared in greater thickness of $97 \mathrm{~mm}$ and compared to the $5-\mathrm{mm}$ sample. Values of $T_{i}$ for $100 \mathrm{~mm}$ for this glass are computed from the values of $T_{\mathrm{i}}$, for $92 \mathrm{~mm}$ so determined.
}

measurements with the thick samples, the compensation beam of the spectrophotometer does not contain a sample of the same length (as it usually does, for example, in measuring the transmittance of a solution relative to its solvent).

Four types of spectrophotometers were available for this work. The designs of two of these instruments (the General Electric recording spectrophotometer and the König-Martens visual spectrophotometer) are such as to give confidence in the results for thick samples. The other two instruments (the Beckman quartz photoelectric spectrophotometer and the Gibson photoelectric spectrophotometer [9] ${ }^{4}$ were sensitive to beam disturbance with thick samples. In the work on the rare-êarth glass 1.673 , it was found that the latter instrument was too sensitive to beam disturbance on insertion of the thick sample to give valid results, and it therefore was not used in the later measurements on the other glasses.

In the General Electric spectrophotometer $(\mathrm{GE})$ $[1,2,3]$ the beam of radiant energy, after transmission through the sample, is incident upon a diffusing white surface of uniform reflectance forming part of the interior wall of a white-lined integrating sphere. Any deviation or dispersion of the beam should not, therefore, cause appreciable error, if not so great that part of the beam impinges on the entrance aperture of the sphere.

On the König-Martens spectrophotometer (K-M) [4] the reverse but equivalent condition exists. The radiant energy is reflected from a diffusing surface of $\mathrm{MgO}$, which is part of an enclosure completely covered with $\mathrm{MgO}$, except for the part containing the bases of the lamps from which the radiant energy originates. Multiple reflections should give a wall surface of essentially uniform radiance, and any displacement of this source in the photometric field by the thick sample should not introduce important error in the results.

The principal value of the K-M measurements was to confirm the reliability of the GE data even for the thickest samples. However, the precision of the visual measurements is less than the photoelectric, and except with the rare-earth glass 1.673 , only a few check measurements were made on the K-M spectrophotometer.

The Beckman quartz photoelectric spectrophotometer (model DU) (BQ) $[5,6]$ is somewhat susceptible to errors resulting from beam disturbance on insertion of the sample, and the thicker the sample the greater may be the error. On this instrument, the

\footnotetext{
Figures in brackets indicate the index references at the end of this paper.
} 
beam of radiant energy, after passage through the sample, is incident directly on the photocell. Although the window of the photocell is made diffusing, this is undoubtedly far from completely so, and any deviation or dispersion of the beam may well affect the response because of varying sensitivity of the photosensitive surface. The principal value of the Beckman spectrophotometer in the present work was to obtain the values of transmittance or internal transmittance in the ultraviolet, and it was not used at wavelengths longer than 460 millimicrons $(m \mu)$.

Consistent with the above, the GE recording spectrophotometer was used over the range 400 to $1,000 \mathrm{~m} \mu$, and the Beckman spectrophotometer over the range from $460 \mathrm{~m} \mu$ toward shorter wavelengths into the ultraviolet as far as there was measurable transmission. The transmittance values obtained on the GE spectrophotometer were taken as a reference and the Beckman values adjusted to them when necessary in accordance with the following procedure: ${ }^{5}(1)$ The GE transmittance values were used from 460 to $1,000 \mathrm{~m} \mu$, subject to an occasional smoothing not exceeding 0.001 ; (2) from 410 to 460 $\mathrm{m} \mu$ the GE data were used to compute an adjustment factor to apply to all of the Beckman data. This adjustment factor was the average of the ratios $\left(T_{\mathrm{GE}} / T_{\mathrm{BQ}}\right)$ at each of the six wavelengths from 410 to $460 \mathrm{~m} \mu$. In this range the adopted values were then based on both the GE data and the adjusted Beckman data; (3) from $400 \mathrm{~m} \mu$ toward shorter wavelengths the adopted values were based on these adjusted Beckman data.

All the values by the Beckman spectrophotometer were obtained with the samples at $25^{\circ} \mathrm{C} .{ }^{6}$ Values by the GE spectrophotometer (visible and infrared) were obtained at a room temperature close to $25^{\circ} \mathrm{C}$, and should be valid also at $25^{\circ} \mathrm{C}$.

\section{Spectrophotometric Results}

Curves of the transmittances for 1-mm thickness for four glasses are shown in figure 1 (numbered 1 to 4 ), together with the transmittance curve for the 5 -mm thickness of the rare-earth glass 1.673 , numbered 5 in the figure.

Curves for the internal transmittances for 4- and $100-\mathrm{mm}$ thicknesses of the same four glasses are shown in figure 2, together with the internal transmittance curve for the $100-\mathrm{mm}$ thickness of the rareearth glass 1.673 .

The values of transmittance and internal transmittance are given in table 1 . All are subject to slight uncertainty in the last figure. Examination of the values of $T_{i}$ for $100 \mathrm{~mm}$ for the rare-earth glass 1.673 shows traces of the absorption bands near 580 and $740 \mathrm{~m} \mu$ commonly found in didymium glasses.

\footnotetext{
${ }_{5}^{5}$ This indicated procedure was followed for 4 of the 5 glasses. For rare-earth glass 1.673 , a slightly different procedure was used.

${ }_{6}$ At the steep portions of the curves (figs. 1 and 2), the transmittance and internal transmittance vary importantly with temperature, in these cases in the ultraviolet region of the spectrum [7].
}

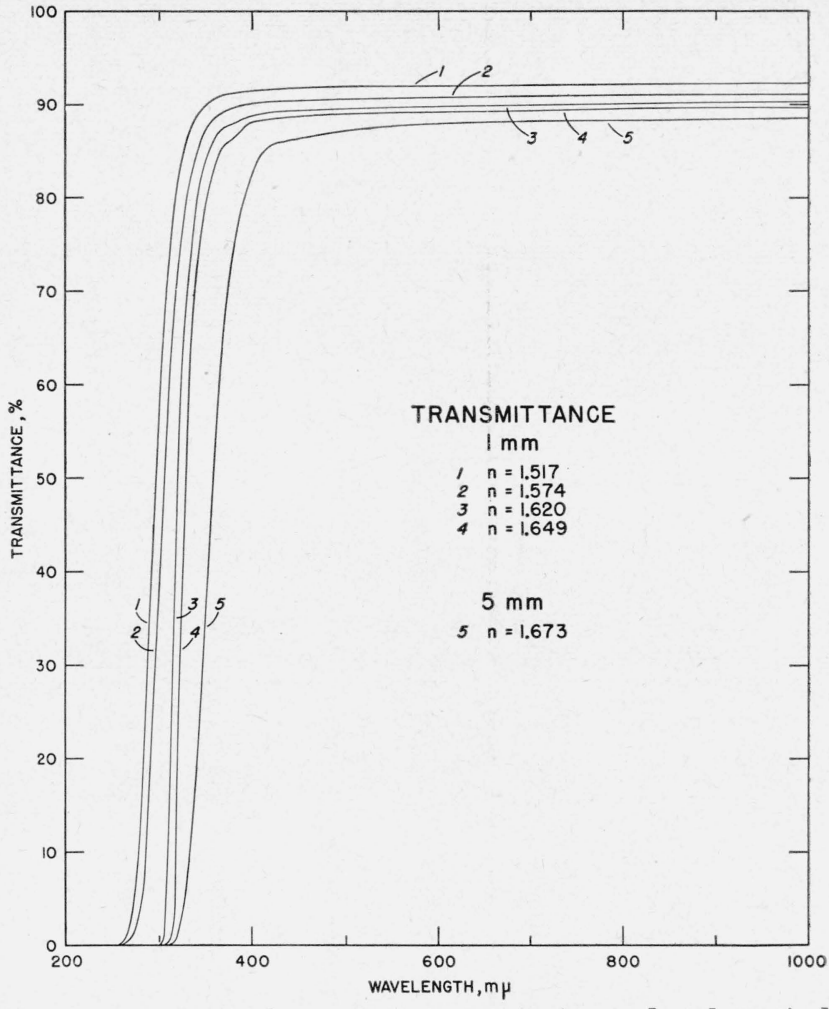

Figure 1. Spectral transmittance of five selected optical glasses made at NBS for the ultraviolet, visible, and nearinfrared spectrum.

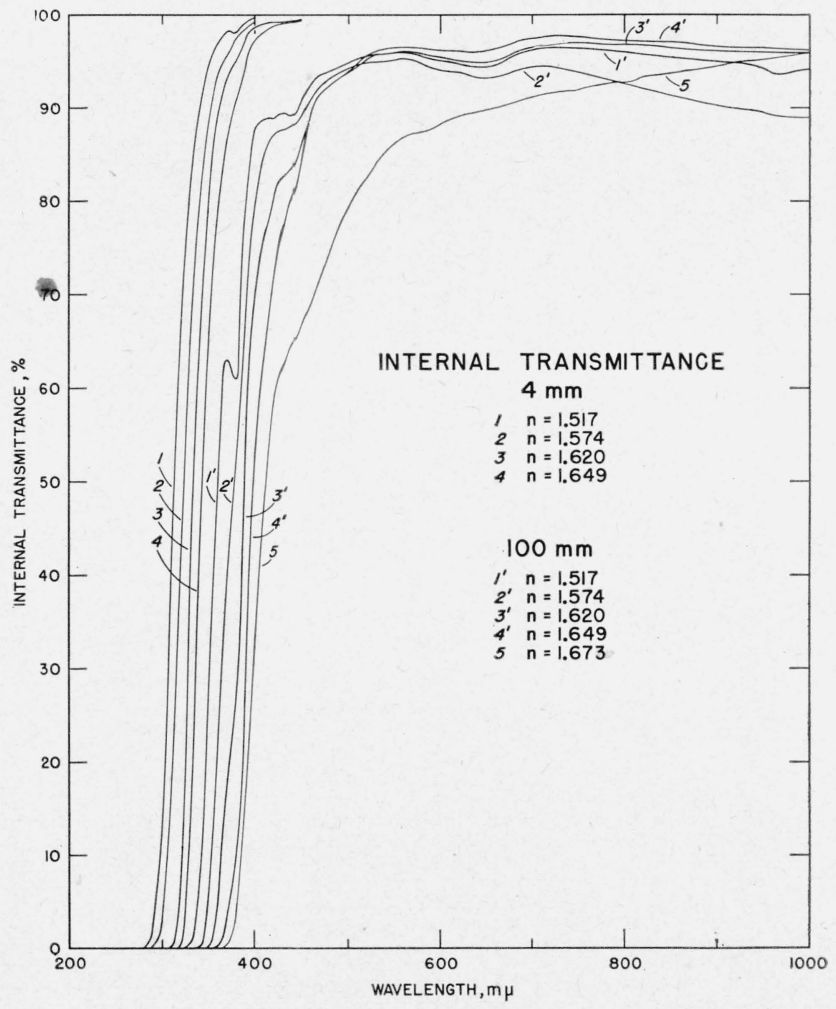

Figure 2. Spectral internal transmittance of five selected optical glasses made at NBS for the ultraviolet, visible, and near-infrared spectrum. 
TABLE 1. Spectral transmittance and internal transmittance data of five selected optical glasses made at NBS for the indicated thicknesses and for the wavelength range 200 to 1,000 millimicrons

\begin{tabular}{|c|c|c|c|c|c|c|c|c|c|c|c|c|c|c|}
\hline \multirow{3}{*}{$\begin{array}{l}\text { Wave- } \\
\text { length }\end{array}$} & \multicolumn{3}{|c|}{$\begin{array}{c}\text { Glass BSC } \\
\begin{array}{c}n=1.517 \\
\nu=64.5\end{array}\end{array}$} & \multicolumn{3}{|c|}{$\begin{array}{c}\text { Glass LBC } \\
\begin{array}{c}n=1.574 \\
\nu \\
\nu=57.7\end{array}\end{array}$} & \multicolumn{3}{|c|}{$\begin{array}{c}\text { Glass F } \\
n=1.620 \\
\nu=36.2\end{array}$} & \multicolumn{3}{|c|}{$\begin{array}{c}\text { Glass DF } \\
n=1.649 \\
\nu=33.8\end{array}$} & \multicolumn{2}{|c|}{$\begin{array}{c}\text { Rare-earth glass } \\
\begin{array}{c}n=1.673 \\
\nu=56.2\end{array}\end{array}$} \\
\hline & $1 \mathrm{~mm}$ & $4 \mathrm{~mm}$ & $100 \mathrm{~mm}$ & $1 \mathrm{~mm}$ & $4 \mathrm{~mm}$ & $100 \mathrm{~mm}$ & $1 \mathrm{~mm}$ & $4 \mathrm{~mm}$ & $100 \mathrm{~mm}$ & $1 \mathrm{~mm}$ & $4 \mathrm{~mm}$ & $100 \mathrm{~mm}$ & $5 \mathrm{~mm}$ & $100 \mathrm{~mm}$ \\
\hline & $T$ & $T_{j}$ & $T_{i}$ & $T$ & $T_{j}$ & $T_{j}$ & $T$ & $T_{j}$ & $T_{j}$ & $T$ & $T_{j}$ & $T_{j}$ & $T$ & $T_{j}$ \\
\hline $\begin{array}{l}m \mu \\
250 \\
260 \\
270 \\
280 \\
290\end{array}$ & $\begin{array}{l}0.000 \\
.002 \\
.026 \\
.12 \\
.33\end{array}$ & $\begin{array}{r}0.000 \\
.001 \\
.019\end{array}$ & 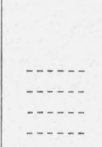 & $\begin{array}{l}0.000 \\
.008 \\
.054 \\
.17\end{array}$ & $\begin{array}{r}0.000 \\
.003\end{array}$ & - & - & .... & -...... & - & & , & $\begin{array}{r}0.000 \\
.001\end{array}$ & \\
\hline $\begin{array}{l}300 \\
310 \\
320 \\
330 \\
340\end{array}$ & $\begin{array}{l}.56 \\
.72 \\
.813 \\
.862 \\
.887\end{array}$ & $\begin{array}{l}.14 \\
.39 \\
.64 \\
.802 \\
.896\end{array}$ & $\begin{array}{l} \\
0.000 \\
.004 \\
.065\end{array}$ & $\begin{array}{l}.37 \\
.56 \\
.71 \\
.80 \\
.847\end{array}$ & $\begin{array}{l}.039 \\
.16 \\
.39 \\
.62 \\
.79\end{array}$ & $\begin{array}{c} \\
0.000 \\
.002\end{array}$ & $\begin{array}{c}0.000 \\
.10 \\
.46 \\
.69 \\
.79\end{array}$ & $\begin{array}{c}0.000 \\
.002 \\
.072 \\
.36 \\
.63\end{array}$ & $\begin{array}{l} \\
\cdots \\
\cdots\end{array}$ & $\begin{array}{l}0.000 \\
.003 \\
.17 \\
.51 \\
.70\end{array}$ & $\begin{array}{l}0.000 \\
.003 \\
.11 \\
.39\end{array}$ & 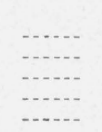 & $\begin{array}{l}.002 \\
.004 \\
.014 \\
.063 \\
.18\end{array}$ & \\
\hline $\begin{array}{l}350 \\
360 \\
370 \\
380 \\
390\end{array}$ & $\begin{array}{l}.900 \\
.907 \\
.910 \\
.911 \\
.913\end{array}$ & $\begin{array}{l}.945 \\
.972 \\
.982 \\
.981 \\
.992\end{array}$ & $\begin{array}{l}.24 \\
.48 \\
.63 \\
.61 \\
.79\end{array}$ & $\begin{array}{l}.873 \\
.886 \\
.893 \\
.896 \\
.899\end{array}$ & $\begin{array}{l}.884 \\
.939 \\
.966 \\
.975 \\
.985\end{array}$ & $\begin{array}{l}.046 \\
.21 \\
.42 \\
.53 \\
.66\end{array}$ & $\begin{array}{l}.840 \\
.864 \\
.876 \\
.879 \\
.885\end{array}$ & $\begin{array}{l}.79 \\
.885 \\
.934 \\
.949 \\
.974\end{array}$ & $\begin{array}{l}.003 \\
.047 \\
.18 \\
.26 \\
.49\end{array}$ & $\begin{array}{l}.79 \\
.832 \\
.857 \\
.864 \\
.874\end{array}$ & $\begin{array}{l}.63 \\
.78 \\
.867 \\
.905 \\
.946\end{array}$ & $\begin{array}{c}0.000 \\
.002 \\
.030 \\
.09 \\
.26\end{array}$ & $\begin{array}{l}.36 \\
.53 \\
.66 \\
.74 \\
.795\end{array}$ & $\begin{array}{l}0.000 \\
.004 \\
.042 \\
.15\end{array}$ \\
\hline $\begin{array}{l}490 \\
410 \\
420 \\
430 \\
440\end{array}$ & $\begin{array}{l}.915 \\
.915 \\
.916 \\
.916 \\
.916\end{array}$ & $\begin{array}{c}.996 \\
-1 \\
-1 \\
\end{array}$ & $\begin{array}{l}.875 \\
.889 \\
.889 \\
.895 \\
.892\end{array}$ & $\begin{array}{l}.901 \\
.902 \\
.902 \\
.903 \\
.903\end{array}$ & \begin{tabular}{c}
.992 \\
\hdashline-1. \\
-
\end{tabular} & $\begin{array}{l}.80 \\
.856 \\
.869 \\
.878 \\
.880\end{array}$ & $\begin{array}{l}.888 \\
.890 \\
.891 \\
.892 \\
.892\end{array}$ & $\begin{array}{l}.987 \\
.990 \\
.991 \\
.993 \\
.993\end{array}$ & $\begin{array}{l}.694 \\
.765 \\
.794 \\
.827 \\
.835\end{array}$ & $\begin{array}{l}.881 \\
.882 \\
.883 \\
.884 \\
.885\end{array}$ & $\begin{array}{l}.971 \\
.981 \\
.986 \\
.990 \\
.992\end{array}$ & $\begin{array}{l}.50 \\
.633 \\
.705 \\
.775 \\
.804\end{array}$ & $\begin{array}{l}.825 \\
.847 \\
.856 \\
.860 \\
.862\end{array}$ & $\begin{array}{l}.32 \\
.47 \\
.575 \\
.625 \\
.645\end{array}$ \\
\hline $\begin{array}{l}450 \\
460 \\
470 \\
480 \\
490\end{array}$ & \begin{tabular}{c}
.917 \\
\hdashline$-\cdot$ \\
\hdashline$-\cdot$
\end{tabular} & 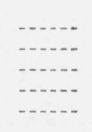 & $\begin{array}{l}.910 \\
.929 \\
.936 \\
.940 \\
.944\end{array}$ & $\begin{array}{c}.904 \\
-\cdots \\
-\end{array}$ & $\begin{array}{l} \\
\cdots\end{array}$ & $\begin{array}{r}.892 \\
.912 \\
.925 \\
.932 \\
.938\end{array}$ & $\begin{array}{c}.893 \\
-\cdots \\
-\cdot- \\
-\cdot-\end{array}$ & 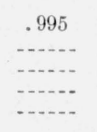 & $\begin{array}{l}.864 \\
.899 \\
.916 \\
.923 \\
.931\end{array}$ & $\begin{array}{c}.887 \\
-\cdots \\
-\cdots \\
-\cdots . . \\
\end{array}$ & \begin{tabular}{c}
.993 \\
\hdashline$-\cdot$ \\
$-\cdots$ \\
$-\cdots$
\end{tabular} & $\begin{array}{l}.842 \\
.900 \\
.913 \\
.923 \\
.932\end{array}$ & $\begin{array}{c}.864 \\
- \\
-\cdots \\
-\cdots\end{array}$ & $\begin{array}{l}.667 \\
.696 \\
.723 \\
.748 \\
.773\end{array}$ \\
\hline $\begin{array}{l}500 \\
510 \\
520 \\
530 \\
540\end{array}$ & $\begin{array}{c}.918 \\
-\cdots \\
-\cdots \\
-\cdots \cdot\end{array}$ & 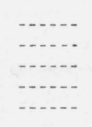 & $\begin{array}{l}.949 \\
.953 \\
.956 \\
.957 \\
.958\end{array}$ & $\begin{array}{c}.905 \\
-1 \\
\end{array}$ & ' & $\begin{array}{l}.942 \\
.946 \\
.948 \\
.949 \\
.950\end{array}$ & $\begin{array}{c}.895 \\
-\cdots \\
-\cdot- \\
-\cdot-\end{array}$ & $\begin{array}{l} \\
\cdots \\
\cdots\end{array}$ & $\begin{array}{l}.938 \\
.947 \\
.952 \\
.956 \\
.958\end{array}$ & $\begin{array}{c}.889 \\
-8 \\
- \\
-\end{array}$ & ב... & $\begin{array}{l}.940 \\
.948 \\
.955 \\
.959 \\
.962\end{array}$ & $\begin{array}{c}.873 \\
-0 \\
\cdots\end{array}$ & $\begin{array}{l}.794 \\
.811 \\
.826 \\
.840 \\
.852\end{array}$ \\
\hline $\begin{array}{l}550 \\
560 \\
570 \\
580 \\
590\end{array}$ & 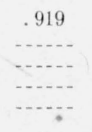 & 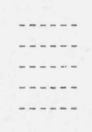 & $\begin{array}{l}.959 \\
.960 \\
.958 \\
.955 \\
.952\end{array}$ & $\begin{array}{c}.906 \\
-1 \\
\end{array}$ & $\begin{array}{l} \\
\cdots\end{array}$ & $\begin{array}{l}.952 \\
.953 \\
.951 \\
.947 \\
.943\end{array}$ & \begin{tabular}{c}
.896 \\
\hdashline-1 \\
$\cdots$
\end{tabular} & $\begin{array}{l}\ldots \\
\cdots \\
\cdots\end{array}$ & $\begin{array}{l}.959 \\
.961 \\
.960 \\
.958 \\
.956\end{array}$ & $\begin{array}{c}.890 \\
-1 . \\
-\cdots \\
---\end{array}$ & ' & $\begin{array}{l}.964 \\
.965 \\
.965 \\
.964 \\
.962\end{array}$ & $\begin{array}{c}.877 \\
-.1 \\
-\cdots \\
-\cdots\end{array}$ & $\begin{array}{l}.862 \\
.869 \\
.873 \\
.874 \\
.878\end{array}$ \\
\hline $\begin{array}{l}600 \\
610 \\
620 \\
630 \\
640\end{array}$ & $\begin{array}{c}.919 \\
-\cdots \\
-\cdots \\
\end{array}$ & $\begin{array}{l}\ldots \\
\\
\cdots\end{array}$ & $\begin{array}{l}.951 \\
.950 \\
.947 \\
.945 \\
.944\end{array}$ & \begin{tabular}{c}
.907 \\
\hdashline \\
\hdashline
\end{tabular} & - & $\begin{array}{l}.941 \\
.939 \\
.938 \\
.935 \\
.933\end{array}$ & $\begin{array}{c}.897 \\
\end{array}$ & $\begin{array}{c} \\
\cdots\end{array}$ & $\begin{array}{l}.955 \\
.953 \\
.951 \\
.949 \\
.949\end{array}$ & $\begin{array}{c}.891 \\
\\
\\
\end{array}$ & - & $\begin{array}{l}.961 \\
.961 \\
.961 \\
.960 \\
.960\end{array}$ & \begin{tabular}{c}
.879 \\
\hdashline \\
-1
\end{tabular} & $\begin{array}{l}.883 \\
.887 \\
.890 \\
.893 \\
.895\end{array}$ \\
\hline $\begin{array}{l}650 \\
660 \\
670 \\
680 \\
690\end{array}$ & $\begin{array}{c}.920 \\
-. \\
-\cdots\end{array}$ & $\begin{array}{l} \\
\end{array}$ & $\begin{array}{l}.944 \\
.946 \\
.951 \\
.956 \\
.960\end{array}$ & $\begin{array}{c}.908 \\
-1 . \\
-\end{array}$ & - & $\begin{array}{l}.932 \\
.933 \\
.935 \\
.940 \\
.943\end{array}$ & $\begin{array}{c}.898 \\
\\
\end{array}$ & 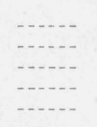 & $\begin{array}{l}.949 \\
.950 \\
.954 \\
.959 \\
.962\end{array}$ & \begin{tabular}{c}
.892 \\
\\
\hdashline \\
\end{tabular} & - & $\begin{array}{l}.961 \\
.962 \\
.966 \\
.969 \\
.973\end{array}$ & \begin{tabular}{c}
.881 \\
\hdashline$-\cdots$ \\
$-\cdots$
\end{tabular} & $\begin{array}{l}.898 \\
.901 \\
.903 \\
.906 \\
.909\end{array}$ \\
\hline $\begin{array}{l}700 \\
710 \\
720 \\
730 \\
740\end{array}$ & $\begin{array}{c}.920 \\
-\cdots \\
-\end{array}$ & מ & $\begin{array}{l}.962 \\
.963 \\
.963 \\
.964 \\
.964\end{array}$ & $\begin{array}{c}.908 \\
-9 \\
-\end{array}$ & - & $\begin{array}{l}.945 \\
.945 \\
.944 \\
.942 \\
.940\end{array}$ & \begin{tabular}{c}
.899 \\
\hdashline-1 \\
\end{tabular} & $\begin{array}{c} \\
\ldots\end{array}$ & $\begin{array}{l}.965 \\
.966 \\
.967 \\
.969 \\
.969\end{array}$ & .893 & - & $\begin{array}{l}.974 \\
.976 \\
.977 \\
.977 \\
.976\end{array}$ & \begin{tabular}{c}
.882 \\
\hdashline-1 \\
\end{tabular} & $\begin{array}{l}.911 \\
.913 \\
.916 \\
.917 \\
.918\end{array}$ \\
\hline $\begin{array}{l}750 \\
760 \\
770 \\
780 \\
790\end{array}$ & $\begin{array}{c}.921 \\
- \\
-\cdot\end{array}$ & ב. & $\begin{array}{r}.963 \\
.963 \\
.963 \\
.962 \\
.961\end{array}$ & $\begin{array}{c}.909 \\
-\cdots \\
\end{array}$ & - & $\begin{array}{l}.938 \\
.936 \\
.934 \\
.931 \\
.929\end{array}$ & \begin{tabular}{c}
.900 \\
\hdashline.. \\
- \\
-
\end{tabular} & 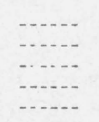 & $\begin{array}{l}.969 \\
.969 \\
.969 \\
.968 \\
.968\end{array}$ & \begin{tabular}{c}
.894 \\
\hdashline-1 \\
-
\end{tabular} & - & $\begin{array}{l}.976 \\
.975 \\
.975 \\
.974 \\
.974\end{array}$ & \begin{tabular}{c}
.882 \\
\hdashline- \\
-
\end{tabular} & $\begin{array}{l}.919 \\
.923 \\
.924 \\
.926 \\
.927\end{array}$ \\
\hline $\begin{array}{l}800 \\
810 \\
820 \\
830 \\
840\end{array}$ & $\begin{array}{c}.921 \\
-0 . \\
-\cdot\end{array}$ & 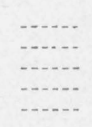 & $\begin{array}{l}.960 \\
.959 \\
.958 \\
.957 \\
.956\end{array}$ & $\begin{array}{c}.909 \\
-9 \\
-\end{array}$ & ב. & $\begin{array}{l}.926 \\
.923 \\
.920 \\
.918 \\
.916\end{array}$ & $\begin{array}{c}.900 \\
\\
\end{array}$ & $\begin{array}{l}\ldots \\
-1\end{array}$ & $\begin{array}{l}.968 \\
.967 \\
.966 \\
.966 \\
.965\end{array}$ & $\begin{array}{c}.895 \\
\\
\end{array}$ & מ & $\begin{array}{l}.973 \\
.972 \\
.972 \\
.972 \\
.971\end{array}$ & $\begin{array}{c}.882 \\
\\
\end{array}$ & $\begin{array}{l}.927 \\
.928 \\
.932 \\
.934 \\
.936\end{array}$ \\
\hline $\begin{array}{l}850 \\
860 \\
870 \\
880 \\
890\end{array}$ & $\begin{array}{c}.922 \\
-9 \\
-\cdots \\
-\cdots\end{array}$ & 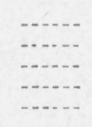 & $\begin{array}{l}.955 \\
.954 \\
.953 \\
.952 \\
.951\end{array}$ & $\begin{array}{c}.909 \\
-\cdots \\
\cdots \\
\end{array}$ & $\begin{array}{l} \\
\ldots \\
\cdots \\
\cdots\end{array}$ & $\begin{array}{l}.913 \\
.911 \\
.909 \\
.907 \\
.905\end{array}$ & 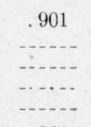 & p. & $\begin{array}{l}.965 \\
.964 \\
.963 \\
.963 \\
.962\end{array}$ & $\begin{array}{c}.896 \\
-.- \\
-\end{array}$ & (n) & $\begin{array}{l}.971 \\
.970 \\
.969 \\
.968 \\
.967\end{array}$ & $\begin{array}{c}.883 \\
-4 \\
-\end{array}$ & $\begin{array}{l}.937 \\
.939 \\
.940 \\
.941 \\
.943\end{array}$ \\
\hline $\begin{array}{l}900 \\
910 \\
920 \\
930 \\
940\end{array}$ & $\begin{array}{c}.922 \\
- \\
-\cdot . \\
\end{array}$ & - & $\begin{array}{l}.950 \\
.949 \\
.948 \\
.947 \\
.946\end{array}$ & $\begin{array}{c}.909 \\
- \\
-\end{array}$ & 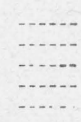 & $\begin{array}{l}.903 \\
.901 \\
.900 \\
.898 \\
.897\end{array}$ & \begin{tabular}{c}
.901 \\
\hdashline \\
\hdashline
\end{tabular} & o. & $\begin{array}{l}.962 \\
.961 \\
.961 \\
.961 \\
.960\end{array}$ & $\begin{array}{c}.896 \\
-0 \\
-\end{array}$ & - & $\begin{array}{r}.966 \\
.966 \\
.965 \\
.965 \\
.964\end{array}$ & \begin{tabular}{c}
.884 \\
\hdashline \\
\end{tabular} & $\begin{array}{l}.945 \\
.947 \\
.948 \\
.950 \\
.952\end{array}$ \\
\hline $\begin{array}{l}950 \\
960 \\
970 \\
980 \\
990\end{array}$ & \begin{tabular}{c}
.922 \\
\hdashline.. \\
$\cdots$ \\
$\cdots$
\end{tabular} & $\begin{array}{l}\ldots \\
\end{array}$ & $\begin{array}{l}.942 \\
.937 \\
.936 \\
.938 \\
.940\end{array}$ & $\begin{array}{c}.909 \\
-1 \\
\end{array}$ & $\begin{array}{l} \\
\end{array}$ & $\begin{array}{l}.895 \\
.891 \\
.890 \\
.890 \\
.890\end{array}$ & .901 & $\begin{array}{c}0 . \\
\ldots\end{array}$ & $\begin{array}{l}.960 \\
.959 \\
.959 \\
.959 \\
.959\end{array}$ & \begin{tabular}{c}
.896 \\
\\
\hdashline \\
\end{tabular} & , & $\begin{array}{l}.964 \\
.963 \\
.962 \\
.962 \\
.962\end{array}$ & $\begin{array}{c}.884 \\
\\
\end{array}$ & $\begin{array}{l}.953 \\
.954 \\
.956 \\
.957 \\
.958\end{array}$ \\
\hline 1000 & .922 & ..... & .941 & .909 & -..... & .890 & .902 & - . & .959 & .896 & - n. & .962 & .885 & .960 \\
\hline
\end{tabular}


Values of $T_{i}$ for $4 \mathrm{~mm}$ are not tabulated above 400 or $450 \mathrm{~m} \mu$ where the values exceed 0.995 . As there is uncertainty of 0.001 or 0.002 in such values, it would be preferable, where the absorption is so slight, to compute the values of $T_{i}$ for $4 \mathrm{~mm}$ (or for any other small thickness) from the values of $T_{i}$ for $100 \mathrm{~mm}$ than to measure them directly.

The slight error in $T_{i}$ resulting from the multiple reflections through different thicknesses of glass [8] is ignored in these results. For either high or low transmittance the error is of the order of 0.001 . In view of the equal or greater uncertainties indicated above, computation of such corrections was considered unwarranted.

The authors express their appreciation to K. S. Gibson, Chief, NBS Photometry and Colorimetry Section, who coordinated the work of these spectrophotometric measurements and results; to Kenneth L. Kelly for his assistance in the computations; and to John C. Schleter for the preparation of the graphs.

\section{References}

[1] A. C. Hardy, History of the design of the recording spectrophotometer, J. Opt. Soc. Am. 28, 360 (1938)

[2] J. L. Michaelson, Construction of the General Electric recording spectrophotometer, J. Opt. Soc. Am. 28, 365 (1938).

[3] K. S. Gibson and H. J. Keegan, Calibration and operation of the General Electric recording spectrophotometer of the National Bureau of Standards, J. Opt. Soc. Am. 28, 372 (1938)

[4] H. J. MeNicholas, Equipment for routine spectral transmission and reflection measurements, BS J. Research 1, 793 (1928) RP30.

[5] H. H. Cary and A. O. Beckman, A quartz photoelectric spectrophotometer, J. Opt. Soc. Am. 31, 682 (1941).

[6] K. S. Gibson and M. M. Balcom, Transmission measurements on the Beckman quarts spectrophotometer, J. Research NBS 38, 601 (1947) RP1798; also J. Opt. Soc. Am. 37, 593 (1947).

[7] K. S. Gibson, Spectrophotometry, NBS Circular 484 (1949). See figures 29 and 30.

[8] T. Smith, Note on measurements of glass absorption, Proc. Phys. Soc. 58, 473 (1946).

[9] K. S. Gibson, Direct-reading photoelectric measurement of spectral transmission, J. Opt. Soc. Am. and Rev. Sci. Instr. \%, 693 (1923).

Washington, November 19, 1953. 